\title{
Determinantes sociais da saúde e prevenção secundária do câncer do colo do útero no Estado do Amazonas, Brasil
}

\author{
Social determinants of health and secondary prevention of cervical cancer in \\ the State of Amazonas, Brazil
}

Juliana Nascimento Viana1, Rosana Pimentel Correia Moysés², Thais Tibery Espir ${ }^{3}$, Gabriela Amaral de Sousa ${ }^{1}$, José Fernando Marques Barcellos ${ }^{4}$, Maria da Graça Pereira Alves ${ }^{5}$

\begin{abstract}
RESUMO
Modelo do estudo: este foi um estudo ecológico transversal e descritivo. Objetivo do estudo: conhecer os determinantes sociais de saúde associados com a prevenção secundária do câncer do colo do útero no período de 2010 a 2014. Metodologia: a unidade amostral foi os 62 municípios do estado do Amazonas. Os dados secundários utilizados foram do Sistema de Informação do Câncer do Colo do Útero (SISCOLO), do Sistema de Informação da Atenção Básica (SIAB), do Instituto Brasileiro de Geografia e Estatística (IBGE) e do Atlas de Desenvolvimento Humano do Brasil. Resultados: baixa cobertura do exame Preventivo do câncer do colo do útero para mulheres de 25 a 64 anos, sendo a faixa etária com menor adesão ao rastreio, a de 60 a 64 anos. A média de analfabetismo feminino na idade de 25 anos ou mais, no Estado, foi de 25,01\%, e este indicador apresentou correlação com a baixa cobertura do exame Preventivo para o câncer do colo do útero em todos os anos. A pouca cobertura da Estratégia Saúde da Família também se associou a baixa adesão ao rastreio. Além disso, a Proporção de Municípios com Amostras Insatisfatórias dos exames citopatológicos apresentou-se acima da meta preconizada pelo Ministério da Saúde. Conclusão: estratégias de promoção da saúde voltadas a adesão ao rastreio devem considerar o nível de escolaridade das mulheres e o fortalecimento da Atenção Primária à Saúde pautada no rastreio não oportunístico; estas devem ser uma das prioridades das políticas públicas nos municípios do Estado.

Palavras-chave: Determinantes Sociais da Saúde. Prevenção Secundária. Neoplasias do Colo do Útero.
\end{abstract}

\section{ABSTRACT}

Study design: This was a cross-sectional and ecological descriptive study. Study objective: Knowing the social determinants of health associated with the secondary prevention of cervical cancer from 2010

1. Graduanda em medicina pela Universidade Federal do Amazonas (UFAM). Pesquisadora de iniciação científica do Núcleo de Atividades Integradas (NAI) da UFAM.

2. Odontóloga. Docente do Dep. de Saúde Coletiva da Faculdade de Medicina da UFAM. Pesquisadora do NAI da UFAM e do grupo de Investigação em Saúde Familiar \& Doença da Universidade do Minho, Portugal.

3. Biomédica. Docente do Dep. de Saúde Coletiva da Faculdade de Medicina da UFAM.

4. Biólogo. Docente do Instituto de Ciências Biológicas da UFAM. Coordenador do NAI da UFAM.

5. Docente Associada com Agregação em medicina pela Universidade do Porto. Docente da Escola de Psicologia da Universidade do Minho, Portugal. Coordenadora do grupo de Investigação em Saúde Familiar \& Doença da Universidade do Minho, Portugal.
CORRESPONDÊNCIA: Juliana Nascimento Viana Universidade Federal do Amazonas Núcleo de Atividades Integradas, Bloco 1 do Instituto de Ciências Biológicas

$2^{\circ}$ andar, sala NAI, Setor Sul. Rua Rodrigo Otávio, Japiim 69000-000 - Manaus/AM - Brasil juliana.nviana@live.com

Recebido em: 30/07/2018 Aprovado em: 24/04/2019 
to 2014. Methods: The sample included the 62 counties of the state of Amazonas, and secondary data were used from the Cervical Cancer Information System, the Primary Care Information System, the Brazilian Institute of Geography and Statistics and the Atlas of Human Development of Brazil. Results: Low coverage of the preventive exam for cervical cancer for women aged 25 to 64 years was found. Also, the age group with the lowest adherence to screening was 60 to 64 years old. The average illiteracy rate among women aged 25 years and over, in the state, was $25.01 \%$, and this indicator correlated with the low coverage of the preventive exam for cervical cancer every year. The low coverage of the Family Health Strategy was also associated with low adherence to screening. The proportion of counties with unsatisfactory samples from the cytopathological examinations was above the goal recommended by the Ministry of Health. Conclusion: Health promotion strategies regarding adherence to screening should consider women's level of education, and the reinforcement of Primary Health Care based on non-opportunistic screening should be one of the priorities of public policies in the counties of the state.

Keywords: Social Determinants of Health. Secondary Prevention. Uterine Cervical Neoplasms.

\section{INTRODUÇÃO}

O Câncer do Colo do Útero (CCU) está entre as principais neoplasias malignas do mundo, sendo a terceira entre as mulheres. Na América Latina, anualmente, são identificados mais de 72 mil novos casos de CCU, representando hoje a principal causa de mortalidade em mulheres na faixa etária de 15 a 44 anos. ${ }^{1}$

Neste contexto, estudos demonstram que nos países como o Brasil, de maior desigualdade social e de população com baixa escolaridade, as mulheres apresentam maior risco de desenvolver o CCU e, devido ao pouco conhecimento dos métodos de rastreamento e a dificuldade de acesso aos serviços de saúde, realizam tardiamente o diagnóstico. ${ }^{2,3}$

A literatura descreve que os determinantes sociais de saúde envolvem uma complexidade de fatores, entre eles as questões sociais, econômicas e de acesso à saúde. Estas condições estão presentes quando analisamos a morbimortalidade por CCU no Brasil, as diferenças parecem resultar de hábitos e comportamentos regionais, decorrentes de um construto social e cultural. 3,4

No Brasil, a previsão para os anos de 2018 e 2019 é de 16.370 novos casos de CCU, representando a terceira neoplasia mais frequente em mulheres e, verdadeiramente, um problema de saúde pública. A maior incidência da doença está na região Norte do país e o estado do Amazonas apresenta o maior número de casos e alta taxa de mortalidade. ${ }^{5,6}$

A ciência mostra que os principais fatores de risco para o CCU são o início precoce da atividade sexual, múltiplos parceiros sexuais, mul- tiparidade, baixa condição socioeconômica, baixo nível de escolaridade, Doenças Sexualmente Transmissíveis (DST), tabagismo e infecção pelo Papilomavírus Humano (HPV). ${ }^{1,6}$

O câncer do colo do útero tem alto potencial de prevenção e quando diagnosticado, precocemente, grandes chances de cura. Hoje, no Brasil, os dois principais mecanismos de prevenção desta doença são a realização do exame citopatológico (Preventivo) em mulheres e a vacinação contra o HPV, em meninas de 9 a 13 anos e rapazes de 12 e 13 anos. ${ }^{1,7}$

Na realidade amazônica, estudos que analisaram o HPV em mulheres habitantes da área rural, principalmente nos chamados municípios ribeirinhos, também apresentam uma prevalência significativa do vírus. No entanto, as moradoras destas localidades apresentam deteç̧ão tardia das lesões precursoras do $\mathrm{CCU}$, sendo que, isto pode ser resultado de fatores, como o pouco conhecimento de medidas preventivas e o acesso limitado aos serviços de saúde. ${ }^{8,9}$

A dificuldade de acesso aos serviços de saúde é uma realidade do Amazonas, sobretudo, devido às suas características geográficas, que já impõem certo isolamento destes municípios ribeirinhos. Outros pontos que influenciam nessa questão são as cheias e vazantes dos rios, a disponibilidade de profissionais e a infraestrutura, esta última, muitas vezes, insuficiente nos serviços de saúde os quais essa população tem acesso. Todos esses obstáculos reforçam a precariedade da Rede de Atenção à Saúde estadual. ${ }^{10,11}$

$\mathrm{O}$ rastreamento do CCU possui diretrizes definidas pelo Ministério da Saúde e tem como méto- 
do a realização do exame preventivo na população alvo, isto é, mulheres na faixa etária de 25 a 64 anos, a cada três anos, após dois exames anuais consecutivos normais. A cobertura preconizada para realização do exame é de $80 \%$ das mulheres. ${ }^{12}$

0 exame preventivo também é avaliado em relação à adequabilidade da amostra citológica. As amostras devem ser satisfatórias com a presença de células representativas da junção escamo-colunar (JEC), pois, este é o local onde se iniciam praticamente todos os cânceres do colo uterino. Esses indicadores deveriam ter suas metas alcançadas em todos os municípios do país. ${ }^{12}$

Deste modo, o monitoramento e avaliação da cobertura do rastreamento do CCU são indicadores da adesão ao rastreio pelas mulheres e da atuação dos municípios na atenção à saúde da mulher. Para isso, o Ministério da Saúde possui o Sistema de Informação do Câncer de Colo de Útero (SISCOLO) que armazena dados referentes aos exames coletados na rede pública de saúde, dados de identificação da mulher; dados demográficos; resultados/laudos dos exames citopatológicos e histológicos; dados sobre a qualidade e quantidade de coletas e seguimento das mulheres. ${ }^{13}$

Sendo assim, sabendo-se que estes dados são importantes ferramentas epidemiológicas e para o planejamento das ações, pois fornecem o panorama da doença no nível local, o objetivo deste estudo é conhecer os determinantes sociais de saúde, associados com a prevenção secundária do CCU, de 2010 a 2014, nos 62 municípios do estado do Amazonas.

\section{METODOLOGIA}

Trata-se de um estudo ecológico transversal, utilizando como unidade amostral os 62 municípios do Amazonas, com base nos dados secundários do Sistema de Informação do Câncer do Colo do Útero (SISCOLO), do Sistema de Informação da Atenção Básica (SIAB), ambos do Ministério da Saúde, do Instituto Brasileiro de Geografia e Estatística (IBGE) e do Atlas de Desenvolvimento Humano do Brasil do Programa das Nações Unidas para o Desenvolvimento (PNUD) do ano de 2010.14,15,16,17

O Quadro 1, descreve as variáveis deste estudo, que possui quatro indicadores sociais, que se referem aos resultados do Atlas de Desenvolvimento Humano do Brasil do ano 2010; um indicador de acesso aos serviços de saúde e quatro indicadores das ações de controle do CCU.

\section{Quadro 1}

Variáveis em análise no estudo

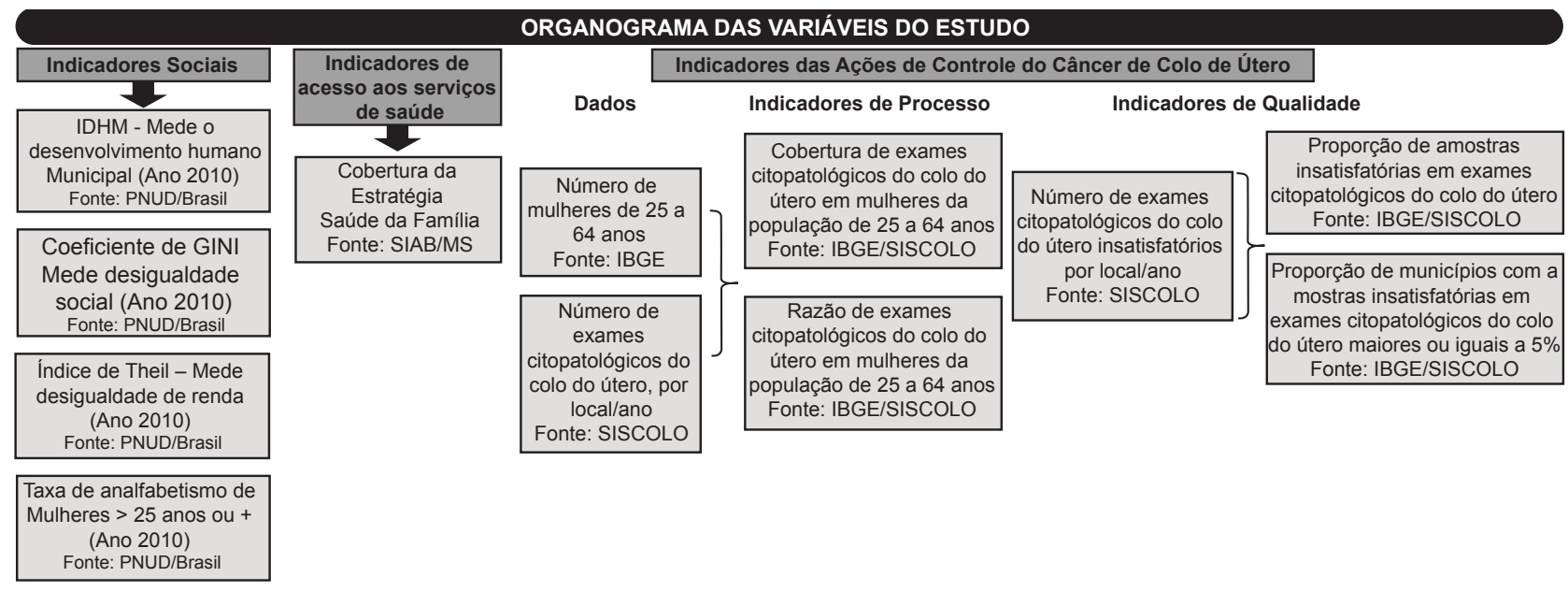

Fonte: Produção dos autores.

Dos indicadores sociais temos o Índice de Desenvolvimento Humano Municipal - IDHM que mede, através das três dimensões: longevidade, educação e renda, o desenvolvimento humano mu- nicipal, variando de 0 a 1 , considerando que, quanto mais próximo de 1 , maior será este indicador. ${ }^{17}$

O Coeficiente de Gini que é usado para estimar a desigualdade social de uma determinada 
população, com dados que variam de 0 a 1 , e quanto mais próximo o valor estiver de 1 , maior é a desigualdade. Já o Índice de Theil mensura a distribuição de renda, considerando a renda familiar per capita, com valores variando, também, de 0 a 1 . Neste indicador, quanto maior o valor, pior a distribuição de renda. Por fim, a Taxa de Analfabetismo em mulheres com 25 anos ou mais, mede o grau de alfabetização destes indivíduos. ${ }^{17}$

O indicador de acesso aos serviços de saúde é a cobertura da Estratégia Saúde da Família (ESF), que significa a proporção da população do município assistida por equipes da ESF. Isso descreve a acessibilidade destas pessoas à Atenção Primária à Saúde, nível de atenção que, na realidade amazônica, é o principal responsável pela realização do exame preventivo para o CCU. ${ }^{15}$

No estudo das variáveis referentes à prevenção secundária do CCU foram utilizados dois indicadores de processo e dois de qualidade. Estes, estão relacionados com a prestação do cuidado a saúde da mulher no controle do CCU e em conformidade com as diretrizes do Ministério da Saúde. ${ }^{12}$

Um dos indicadores de processo foi a cobertura anual de exames citopatológicos do colo do útero, na faixa etária de 25 a 64 anos, pois avalia o alcance do preventivo na população alvo, em um determinado ano e local, através da divisão do número de exames citopatológicos do colo do útero e o número de mulheres nesta faixa etária. ${ }^{12}$

O outro foi a Razão de Cobertura de exames citopatológicos que é um instrumento de análise da realização de um exame preventivo a cada três anos, a partir do mesmo cálculo da cobertura anual, mas considerando somente $1 / 3$ da população de 25 a 64 anos, em determinado ano e local. ${ }^{12}$

Com relação aos indicadores de qualidade, foi analisado o indicador Proporção de Amostras Insatisfatórias de exames citopatológicos do colo do útero, que é o percentual de amostras insatisfatórias do total de exames realizados em determinado local e período. O outro indicador foi Proporção de municípios com amostras insatisfatórias em exames citopatológicos do colo do útero maiores ou iguais a $5 \%$ que demonstra o percentual de municípios do estado com falhas neste procedimento. Esses dois indicadores permitem avaliar a adequação ao acesso à saúde e a Rede Assistencial dos Municípios. ${ }^{12}$
Os dados foram primeiramente descritos e organizados no software Excel $®$, utilizando sua planilha eletrônica para armazenamento e, posteriormente, foi executada a análise de informações, através do programa IBM Statistical Package for the Social Science (SPSS), versão 22.0. Foi realizada a estatística descritiva (médias e frequências) e estimaram-se os coeficientes de correlação de Pearson, para analisar a correlação entre os indicadores sociais, de acesso à saúde e de ações de controle do CCU (prevenção secundária).

\section{RESULTADOS}

A cobertura do exame Preventivo para - CCU foi menor nas mulheres da faixa etária de 60 a 64 anos, em todos os anos avaliados. As maiores coberturas do Preventivo em 2010 (13,64\% DP $\pm 4,19), 2011(17,78 \% \mathrm{DP} \pm 10,43), 2013$ $(18,83 \%$ DP $\pm 10,64)$ e $2014(22,79 \%$ DP $\pm 12,44)$ foram nas mulheres com intervalos de idade entre 35 e 39 anos e, no ano de $2012(20,71 \%$ DP \pm 9,72), naquelas de 55 a 59 anos.

A Tabela 1 apresenta os resultados dos indicadores sociais e de acesso aos serviços de saúde. Os resultados do IDHM demonstram que os municípios do Amazonas apresentam valor médio. A análise dos indicadores de desigualdade social (Gini e Theil) comprova que estes lugares apresentam um quadro desfavorável de distribuição de renda e assimetria social. ${ }^{17}$

O município do estado do Amazonas que apresentou a menor taxa de analfabetismo em mulheres de 25 anos ou mais teve uma taxa de $4,76 \%$, e o município com maior taxa estava acima dos $48 \%$ desta população. ${ }^{17}$

Ao avaliar a Cobertura da Estratégia Saúde da Família (ESF) no período de 2010 a 2014, houve um aumento gradativo, mas sem alcançar $100 \%$ de cobertura da ESF em todos os municípios. ${ }^{15}$

A Tabela 2 apresenta os indicadores das ações de controle do CCU. Relativamente aos indicadores de processo, a Cobertura do exame Preventivo nos municípios foi abaixo de $60 \%$ em todos os anos analisados, tendo, no máximo, um alcance de cerca de $50 \%$. A Razão de Cobertura também se apresentou abaixo da meta que é 1 , nos anos analisados, confirmando que todas as cidades não atingiram as diretrizes propostas pelo Ministério da Saúde. ${ }^{12}$ 
Os resultados dos indicadores de qualidade demonstram que a proporção de amostras insatisfatórias não corresponde as orientações preconizadas pelo Ministério da saúde, que é abaixo do valor 1 . No entanto, vale ressaltar que cerca de $20 \%$ dos municípios do Estado não possuem informações referentes a estas amostras para que a mensuração seja feita concretamente. ${ }^{12}$

\section{Tabela 1}

Caracterização dos Indicadores dos Determinantes Sociais de Saúde do estado do Amazonas

\begin{tabular}{|c|c|c|c|c|c|}
\hline Indicadores & $\mathrm{N}^{*}$ & Mínimo & Máximo & Média & Desvio Padrão \\
\hline IDH Municipal (Ano 2010) & 62 & 0,450 & 0,737 & 0,565 & 053 \\
\hline Coeficiente de Gini (Ano 2010) & 62 & 0,52 & 0,80 & 0,619 & 058 \\
\hline Índice de Theil (Ano 2010) & 62 & 0,46 & 1,28 & 0,669 & 0,14 \\
\hline Taxa de Analfabetismo (mulheres $>25$ anos ou + ) & 62 & 4,76 & 48,14 & 25,01 & 11,17 \\
\hline Cobertura da ESF/Ano 2010 & 62 & 13,58 & 100 & 70,33 & 26,40 \\
\hline Cobertura da ESF/Ano 2011 & 62 & 0,00 & 100 & 68,55 & 27,66 \\
\hline Cobertura da ESF/Ano 2012 & 62 & 13,35 & 100 & 68,90 & 25,74 \\
\hline Cobertura da ESF/Ano 2013 & 62 & 13,30 & 100 & 70,19 & 23,69 \\
\hline Cobertura da ESF/Ano 2014 & 62 & 35,21 & 100 & 85,50 & 17,64 \\
\hline
\end{tabular}

*N representa o número de municípios.

Fonte: PNUD/IBGE/SIAB.

\section{Tabela 2}

Caracterização dos Indicadores das Ações de controle do Câncer de Colo de Útero do estado do Amazonas

\begin{tabular}{|c|c|c|c|c|c|}
\hline \multicolumn{6}{|c|}{ Indicadores de Processo } \\
\hline Ano & $\begin{array}{c}2010 \\
\text { (Média + DP) }\end{array}$ & $\begin{array}{c}2011 \\
\text { (Média + DP) }\end{array}$ & $\begin{array}{c}2012 \\
\text { (Média + DP) }\end{array}$ & $\begin{array}{c}2013 \\
\text { (Média + DP) }\end{array}$ & $\begin{array}{c}2014 \\
\text { (Média + DP) }\end{array}$ \\
\hline \multirow{2}{*}{$\begin{array}{l}\text { Cobertura anual do } \\
\text { exame Preventivo }\end{array}$} & $\mathrm{N}=62$ & $N=62$ & $N=62$ & $N=62$ & $N=62$ \\
\hline & $12,30(+5,43)$ & $16,13(+8,76)$ & $16,84(+7,76)$ & $16,44(+8,86)$ & $19,94(+10,49)$ \\
\hline \multirow{2}{*}{$\begin{array}{l}\text { Razão de Cobertura do } \\
\text { exame Preventivo }\end{array}$} & $N=62$ & $N=62$ & $N=62$ & $N=62$ & $N=62$ \\
\hline & $0,36(+0,16)$ & $0,48(+0,26)$ & $0,50(+0,23)$ & $0,49(+0,26)$ & $0,59(+0,31)$ \\
\hline \multicolumn{6}{|c|}{ Indicadores de Qualidade } \\
\hline Ano & $\begin{array}{c}2010 \\
\text { (Média + DP) }\end{array}$ & $\begin{array}{c}2011 \\
\text { (Média + DP) }\end{array}$ & $\begin{array}{c}2012 \\
\text { (Média + DP) }\end{array}$ & $\begin{array}{c}2013 \\
\text { (Média + DP) }\end{array}$ & $\begin{array}{c}2014 \\
\text { (Média + DP) }\end{array}$ \\
\hline \multirow{2}{*}{$\begin{array}{l}\text { Proporção de Amostras } \\
\text { Insatisfatórias } * *\end{array}$} & $N=52$ & $N=49$ & $N=49$ & $N=49$ & $N=50$ \\
\hline & $4,81(+13,51)$ & $1,73(+2,61)$ & $2,25(+3,19)$ & $4,44(+4,46)$ & $3,24(+2,95)$ \\
\hline \multirow{2}{*}{$\begin{array}{l}\text { Proporção de Municípios } \\
\text { com Amostras Insatis- } \\
\text { fatórias > ou = a } 5 \%\end{array}$} & $N=62$ & $N=62$ & $N=62$ & $N=62$ & $N=62$ \\
\hline & $16,12 \%$ & $6,45 \%$ & $8,06 \%$ & $27,41 \%$ & $22,58 \%$ \\
\hline
\end{tabular}

${ }^{* *} \mathrm{~N}$ representa o número de municípios que tinham essa informação no SISCOLO.

Fonte: IBGE/SISCOLO.

O outro indicador de qualidade, a Proporção de Municípios que apresentaram amostras insatisfatórias em exames citopatológicos do colo do útero, sendo estas, maiores ou iguais a $5 \%$, possui dados mais fiáveis e tem um percentual acima da meta em todos os anos analisados para o estado do Amazonas. ${ }^{12}$
Os resultados da Tabela 3 apresentam as correlações, excetuando-se o indicador de Razão de Cobertura, pois ele avalia a realização do exame pela população alvo a cada três anos, e o objeto desta análise é caracterizar os fatores associados com prevenção secundária anualmente. 


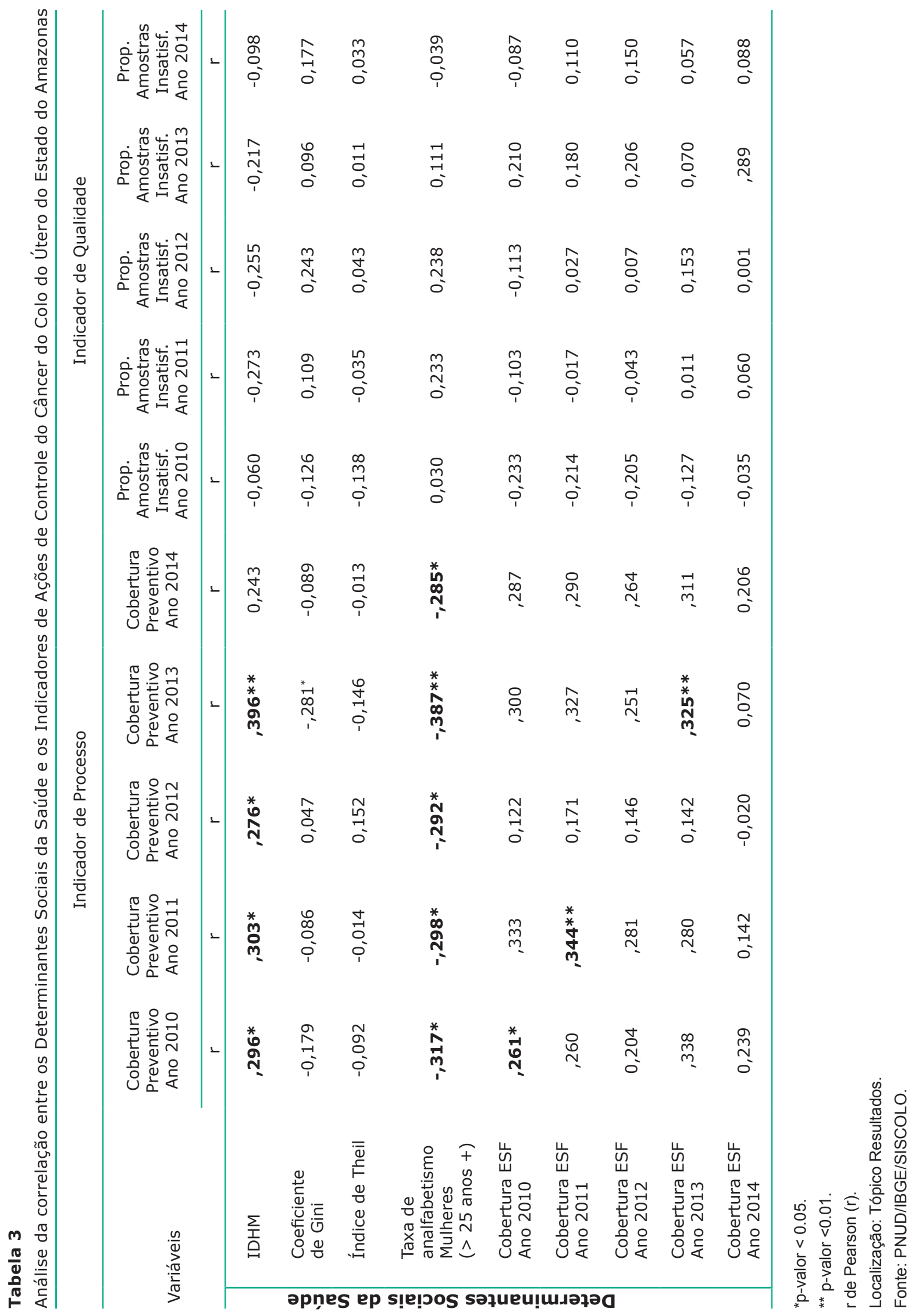


Os resultados demonstram uma associação positiva entre a cobertura do exame Preventivo e o IDHM. Isto significa que quanto maior o Índice de Desenvolvimento Humano, maior a cobertura deste exame. ${ }^{17}$

O Índice de Theil não está associado, significativamente, aos resultados de Cobertura do Preventivo. Deste modo, a distribuição de renda no caso do estado do Amazonas não é um indicador que impacta nos indicadores de processo e na qualidade das ações de controle do $\mathrm{CCU} \cdot{ }^{17}$

A Taxa de Analfabetismo em mulheres de 25 anos ou mais teve uma associação negativa com a Cobertura do Preventivo, em todos os anos analisados, inferindo, assim, que quanto maior o analfabetismo, pior é a adesão ao rastreio do CCU. ${ }^{17}$

\section{DISCUSSÃO}

Segundo dados da Fundação Centro de Controle de Oncologia do Estado do Amazonas, o CCU é a neoplasia com maior morbimortalidade em mulheres amazônicas. No período de análise deste estudo, 2011 a 2014, morreram em média 198 mulheres por ano neste hospital devido a doença. Estes dados reiteram a importância de estratégias de prevenção e tratamento mais efetivos e pressupõe o impacto social e familiar desta doença para o estado. ${ }^{18,19,20,21}$

Este estudo demonstrou que os dois principais indicadores de processo nas Ações de controle do CCU - Cobertura e Razão de cobertura - estão abaixo das metas preconizadas pelo Ministério da Saúde em todos os anos analisados e que a faixa etária de 60 a 64 anos é que tem a menor a adesão ao rastreio para o CCU. A faixa etária de 30 a 35 anos é a que tem a maior cobertura do exame. Estes resultados corroboram outros estudos realizados na Região amazônica, onde as mulheres mais velhas também apresentaram as menores coberturas e razões de cobertura, o que significa que essas mulheres não realizaram o exame preventivo para o CCU anualmente e não o fazem a cada 3 anos. ${ }^{22,23}$

Estudos apontam que os principais motivos para a não realização do Preventivo em mulheres da região Amazônica são: a pouca informação sobre o exame; sentimentos de medo e vergonha; a demora no resultado; a falta de interesse e tempo para a realização e a dificuldade de acesso ao serviço de saúde. Associados a esses fatores, temos indicadores sociais importantes, como o fato dessas pessoas, em sua maioria, serem donas de casa, possuírem baixa escolaridade e baixo nível socioeconômico. 22,24

No estado do Amazonas, apesar da prevalência de um IDHM médio nos municípios, temos cidades com baixo IDHM. Este indicador social se correlacionou com a cobertura do exame preventivo em todos os anos analisados, o que expressa que quanto melhor o desenvolvimento humano municipal maior a cobertura do exame preventivo. ${ }^{17}$

O estudo de Sadovsky et al. (2015) ${ }^{25}$ também evidenciou uma forte correlação entre este indicador social e a adesão a prevenção secundária para o $\mathrm{CCU}$, podendo inferir que melhorias nos três fatores de análise do IDHM, sendo eles, a expectativa de vida ao nascer, a educação e o Produto Interno Bruto (PIB) per capita, podem gerar um aumento nos indicadores de prevenção secundária e reduzir a alta morbimortalidade pela doença.

Os resultados dos indicadores sociais deste estudo são consoantes aos da pesquisa de Viana, Freitas e Giatti (2016) ${ }^{26}$, que demonstrou o fato de o Amazonas, no período de 2002 a 2011, ser o estado da Amazônia Legal a ter o pior desempenho no aumento do Produto Interno Bruto. Isto significa menores atividade econômica, produção, consumo e investimento, podendo se justificar, deste modo, os piores indicadores sociais.

Os mesmos autores descrevem o Amazonas como um dos estados que apresentou os piores indicadores relacionados a saneamento básico, mortalidade por doenças infecciosas e parasitárias, insegurança alimentar e desnutrição infantil, sendo, todos esses, relevantes para análise da morbimortalidade da população. ${ }^{26}$

Em nosso estudo, assim como descrito na literatura, a Taxa de Analfabetismo em mulheres com 25 anos ou mais, associou-se às baixas coberturas do Preventivo, isto é, quanto maior o analfabetismo, menor foi a realização do exame.

Os resultados deste indicador são inquietantes, pois a média foi de $25,01 \%$ para o Estado, sendo cinco vezes mais alta do que o nível 
aceitável pela Organização das Nações Unidas para a Educação, a Ciência e a Cultura (UNESCO), que determina que taxas de analfabetismo acima de $5 \%$ são inaceitáveis. Este é um problema real, reiterando que a baixa escolaridade deve ser um dos principais fatores a serem considerados na construção de estratégias, a fim de intensificar a realização do exame pelas mulheres amazônicas. 22,24,27

Os estudos com populações socialmente vulneráveis também reconheceram que a baixa escolaridade é um desafio para a prevenção secundária do CCU, sobretudo, por afetar diretamente a compreensão das mulheres sobre o exame e sua importância para saúde. ${ }^{24}$

Neste sentido, experiências bem-sucedidas decorrem do uso de instrumentos inovadores de educação em saúde, com enfoque no CCU, como o estudo de Abiodun et al. (2014) ${ }^{28}$, que utilizaram uma intervenção audiovisual abordando o exame Preventivo, com a finalidade aumentar 0 entendimento das mulheres nigerianas de comunidades rurais. Os resultados demonstraram um aumento de $50 \%$ na adesão ao rastreio, sendo que, com relação à avaliação do conhecimento sobre o CCU e exames, ocorreu um aumento de $2 \%$ para $70,5 \%$.

Os indicadores de qualidade não se associaram aos determinantes sociais de saúde em análise neste estudo. No entanto, cabe ressaltar como dificuldade encontrada no estado do Amazonas, o registro dos dados referentes às amostras insatisfatórias dos exames citopatológicos, pois em todos os anos avaliados, cerca de $20 \%$ dos municípios não tinham informações registradas no SISCOLO. ${ }^{12}$

Sendo assim, não podemos fazer inferência concreta sobre esse resultado. Contudo, a análise do outro indicador de qualidade, a Proporção de Municípios com Amostras Insatisfatórias, apresentou-se acima da diretriz do Ministério da Saúde, salientando que os indicadores de qualidade devem ser umas das prioridades de atuação das políticas de saúde no Estado. ${ }^{12}$

Os estudos que avaliaram o indicador de Proporção de Municípios com Amostras Insatisfatórias constataram que a região Norte do país apresentou os piores percentuais, principalmente na faixa etária de 60 a 64 anos. Entretanto, o
Amazonas exibiu resultados dentro das diretrizes do Ministério da Saúde, com base na análise dos municípios com informações registradas no SISCOLO. $27,29,30$

Deste modo, convém dizer que a implantação do Programa Viva Mulher no Estado, em 1999, teve impacto positivo nos anos seguintes, nos indicadores de qualidade, em virtude da capacitação dos profissionais no recolhimento e armazenamento das lâminas de citopatológico. Isto confirma que ciclos de capacitação mais frequentes poderiam melhorar os resultados destes indicadores. ${ }^{27,29,30}$

O indicador de acesso aos serviços de saúde, isto é, a cobertura da Estratégia Saúde da Família (ESF), esteve associada com a Cobertura do exame Preventivo e a Razão de Cobertura, nos anos de 2010, 2011 e 2013, reforçando, assim, que o fortalecimento e ampliação do acesso à Atenção Primária à Saúde melhoraria a prevenção primária do CCU. Esse dado se opõe aos resultados de outros estudos que não identificaram essa associação. $O$ fato do exame Preventivo para o CCU no Amazonas ser realizado, majoritariamente, pelas unidades básicas de saúde da ESF pode ser a principal explicação deste resultado. ${ }^{10,26}$

Vale destacar que estudos comprovam que os programas de rastreio do CCU mais eficazes são os organizados em uma rede que define a idade de início ideal, cujas práticas profissionais são pautadas na triagem organizada e as ações do programa respeitam a realidade daquela população, mas sempre considerando que a vacinação contra infecções por HPV é uma prioridade como estratégia de redução da incidência de lesões pré-cancerosas e que o rastreio também é crucial como estratégia preventiva. ${ }^{31,32}$

O estudo de Basu et al. (2018) (3) $^{33}$ avaliar os progressos de 2007 a 2016 na implantação dos protocolos, da organização dos sistemas de saúde em base populacional e uso de carta convite para o rastreio do câncer de mama, colorretal e do colo do útero em países da União Europeia, concluiu que para o câncer de colo do útero os programas foram implantados mais precocemente nos países membros, mas que a conversão para carta-convite baseada na população-alvo ainda é um desafio, mesmo assim ao analisar a evolução no período de 10 anos do rastreamento do câncer do colo do 
útero, houve um aumento de $11,9 \%$ da população que teve acesso ao exame.

A literatura descreve bons resultados com estratégias de convite/convocatória para o rastreio do câncer de colo de útero, como o estudo caso-controle realizado em 13 unidades de cuidados primários em saúde de Portugal que comparou o uso de carta escrita com envio de mensagens de texto (SMS) e ligações telefônicas como convocação para realização do exame preventivo para o câncer do colo de útero, e concluiu que com a utilização do contato telefônico (SMS/ligação) foi mais eficaz, representando $13,3 \%$ maior adesão ao rastreio neste grupo em comparação com o grupo convidado por carta escrita. ${ }^{34}$

Por fim, considerando a realidade amazônica, com suas características peculiares, principalmente nas áreas rurais e/ou comunidades ribeirinhas, cabe descrever o estudo de Erwin et al. (2019) realizado na Tanzânia em populações rurais e ribeirinhas, que considerou a importância da acessibilidade, do custo de transporte e da compreensão da importância do exame preventivo para câncer do colo de útero na formulação da estratégia de convite. As mulheres foram divididas em três grupos, em que o grupo controle não recebia nenhum convite, um grupo recebia mensagens de texto por telefone (SMS) baseadas no modelo de Crenças de doença com enfoque na prevenção do câncer de colo de útero, o grupo da intervenção recebia as mensagens de texto e um voucher de transporte. Os resultados demonstraram que o grupo que recebeu o voucher teve maior adesão ao rastreio, comprovando que o acesso é um limitador de realização do exame. ${ }^{35}$

\section{CONCLUSÃO}

Os resultados deste estudo reiteram que estratégias de promoção da saúde voltadas a adesão ao rastreio, devem considerar o baixo nível de escolaridade das mulheres e, por isso, precisam ser desenvolvidas em uma perspectiva que corresponda a literacia em saúde desta população.

Neste sentido, os autores deste estudo desenvolveram uma intervenção audiovisual baseada nas características sociais, culturais e educacionais das mulheres amazônicas, na busca a fim de que os resultados tenham impacto no conhecimento sobre o exame Preventivo para o $\mathrm{CCU}$ e a respeito da doença. ${ }^{36}$

Por fim, o fortalecimento da Atenção Primária à Saúde e a construção de uma rede organizada de rastreio do $\mathrm{CCU}$, pensando em estratégias sensíveis à realidade amazônica para convocação das mulheres, criando um sistema não oportunístico, deveria ser uma das prioridades das políticas públicas nos municípios do Estado, haja vista essa ser a porta de entrada das mulheres no Sistema de Saúde, bem como o principal espaço para a realização de ações de educação em saúde e do exame Preventivo para o CCU.

\section{FINANCIAMENTO}

Este trabalho teve Financiamento da Pró-Reitoria de Pesquisa e Pós-Graduação da Universidade Federal do Amazonas pelo Programa de Apoio à Iniciação Científica do Amazonas (PIBIC) da Universidade Federal do Amazonas (Edital PIBIC/2017).

\section{REFERÊNCIAS}

1. World Health Organization. Sexually transmitted infections (STIs) [internet]. Geneva: World Health Organization; 2016 [acesso em 28 jul 2018]. Disponível em: http://www.who.int/mediacentre/factsheets/fs110/en/

2. Barros Junior J, Freitas KM, Silva VKG, Duarte RB, CarvaIho EMR. O câncer do colo do útero: um rastreamento nos sistemas de informações. Revista Interdisciplinar Encontro das Ciências. 2018;1(1):108-122. Disponível em: http:// www.fvs.edu.br/riec/index.php/riec/article/view/12/9

3. Wünsch Filho V, Antunes JLF, Boing AF, Lorenzi RL. Perspectivas da investigação sobre determinantes sociais em câncer. Physis. 2008;18(3):427-50. Disponível em: http://www.scielo.br/pdf/physis/v18n3/v18n3a04.pdf

4. Discacciati MG, Barboza BMS, Zeferino LC. Por que a prevalência de resultados citopatológicos do rastreamento do câncer do colo do útero pode variar significativamente entre duas regiões do Brasil? Rev Bras Ginecol Obstet. 2014;36(5):192-7. Disponível em: http://www.scielo. br/pdf/rbgo/v36n5/0100-7203-rbgo-36-05-00192.pdf

5. Governo do Estado do Amazonas. Relatórios de gestão [internet]. Fundação Centro de Controle de Oncologia do Estado do Amazonas; 2017. Disponível em: http:// www.fcecon.am.gov.br/institucional/acesso-a-informacao/relatorios-de-gestao/ 
6. INCA. Instituto Nacional do Câncer. Incidência de Câncer no Brasil. Rio de Janeiro: 122 p.2016. Disponível em: http://santacasadermatoazulay.com.br/wp-content/ uploads/2017/06/estimativa-2016-v11.pdf

7. Schiffman M, Saraiya M. Control of HPV-associated cancers with HPV vaccination. Lancet Infect Dis. 2017;17(1):6-8.

8. Pinto DS, Fuzii HT, Quaresma JAS. Prevalência de infecção genital pelo HPV em populações urbana e rural da Amazônia Oriental Brasileira. Cad Saude Publica. 2011;27(4):769-78. Disponível em: http://www.scielo.br/pdf/csp/v27n4/16.pdf

9. Pinheiro MCN, Brito EB, Pinheiro DN, Borges BES, Soares ES, Gomes JAN, et al. Principais genótipos de papilomavírus humano em mulheres ribeirinhas na amazônia brasileira. Rev Bras Promoç Saúde. 2016;29(1):68-74. Disponível em: https://periodicos.unifor.br/RBPS/article/view/4150/pdf

10. Oliveira HM, Gonçalves MJF, Pires ROM. Caracterização da estratégia saúde da família no estado do Amazonas, Brasil: análise da implantação e impacto. Cad Saúde Pública. 2011; 27(1):35-45. Disponível em: http://www.scielo.br/pdf/csp/v27n1/04.pdf

11. Garnelo L, Sousa ABL, Silva CO. Regionalização em Saúde no Amazonas: avanços e desafios. Cien Saude Colet. 2017;22(4):1225-34. Disponível em: http://www.scielo. br/pdf/csc/v22n4/en_1413-8123-csc-22-04-1225.pdf

12. Ministério da Saúde. Controle dos cânceres do colo do útero e da mama [internet]. Brasília: Editora do Ministério da Saúde; 2013. Disponível em: http://bvsms.saude.gov.br/ bvs/publicacoes/controle_canceres_colo_utero_2013.pdf

13. Ministério da saúde. Sistemas de informação do controle do câncer de mama (SISMAMA) e do câncer do colo do útero (SISCOLO): manual gerencial [internet]. Rio de Janeiro: INCA; 2011. Disponível em: http://bvsms. saude.gov.br/bvs/publicacoes/inca/Sistema_de_informacao_do_controle_do_cancer_de_mama.pdf

14. DATASUS. Sistema de Informação do câncer do colo do útero e Sistema de Informação do câncer e mama [internet]. Departamento de Informática do SUS; 2018 [acesso em 10 maio 2018]. Disponível em: http://w3.datasus.gov.br/siscam/index.php?area $=0401$

15. DATASUS. SIAB - Sistema de Informação de Atenção Básica [internet]. Departamento de Informática do SUS; 2016 [acesso em 20 maio 2018]. Disponível em: http://datasus. saude.gov.br/sistemas-e-aplicativos/epidemiologicos/siab

16. Instituto Brasileiro de Geografia e Estatística. Dados do Censo 2010 Publicados no Diário Oficial da União [Internet]. Brasília (DF): IBGE; 2010. [acesso em 20 maio 2018]. Disponível em: https://www.ibge.gov.br/

17. Atlas do Desenvolvimento Humano no Brasil [internet]. 2013. [acesso em 30 maio 2018]. Disponível em: http://atlasbrasil.org.br/2013/

18. Governo do Estado do Amazonas. Prestação de contas ordinária anual: relatório de gestão do exercício de 2011 [internet]. Manaus: Fundação Centro de Controle de Oncologia do Estado do Amazonas; 2011. Disponível em: http://www.fcecon.am.gov.br/wp-content/uploads/2014/08/Relatorio-2011.pdf

19. Governo do Estado do Amazonas. Prestação de contas ordinária anual: relatório de gestão 2012 [internet]. Manaus: Fundação Centro de Controle de Oncologia do Estado do Amazonas; 2013. Disponível em: http://www.fcecon.am. gov.br/wp-content/uploads/2014/08/Relatorio-2012.pdf

20. Governo do Estado do Amazonas. Prestação de contas ordinária anual: relatório anual de gestão 2013 [internet]. Manaus: Fundação Centro de Controle de Oncologia do Estado do Amazonas; 2014. Disponível em: http://www. fcecon.am.gov.br/wp-content/uploads/2014/08/Relatorio-2013.pdf

21. Governo do Estado do Amazonas. Prestação de contas ordinária anual: relatório anual de gestão 2014 [internet]. Manaus: Fundação Centro de Controle de Oncologia do Estado do Amazonas; 2015. Disponível em: http://www. fcecon.am.gov.br/wp-content/uploads/2014/08/Relatorio-2014.pdf

22. Borges MFSO, Dotto LMG, Koifman RJ, Cunha MA, Muniz PT. Prevalência do exame preventivo de câncer do colo do útero em Rio Branco, Acre, Brasil, e fatores associados à não-realização do exame. Cad Saude Publica. 2012; 2899(6):1156-66. Disponível em: http://www.scielo.br/pdf/csp/v28n6/14.pdf

23. Rocha SMM, Bahia MO, Rocha CAM. Perfil dos exames citopatológicos do colo do útero realizados na Casa da Mulher, Estado do Pará, Brasil. Rev Pan-Amaz Saude. 2016;7(3):51-5. Disponível em: http://scielo.iec.gov. br/pdf/rpas/v7n3/2176-6223-rpas-7-03-00051.pdf

24. Leite SM. Fatores relacionados a não adesão ao preventivo de câncer de colo uterino em Parintins Amazonas [Trabalho de Conclusão de Curso]. Parintins (AM): Universidade do Estado do Amazonas; 2017.

25. Sadovsky ADI, Poton WL, Reis-Santos $B$, Barcelos MRB, Silva ICM. Índice de Desenvolvimento Humano e prevenção secundária de câncer de mama e colo do útero: um estudo ecológico. Cad Saude Publica. 2015; 31(7):1539-50. Disponível em: http://www. scielo.br/pdf/csp/v31n7/0102-311X-csp-31-7-1539.pdf

26. Viana RL, Freitas CM, Giatti LL. Saúde ambiental e desenvolvimento na Amazônia legal: indicadores socioeconômicos, ambientais e sanitários, desafios e perspectivas. Saude Soc . 25(1):233-46. Disponível em: http://www.scielo. br/pdf/sausoc/v25n1/1984-0470-sausoc-25-01-00233.pdf

27. Nobre JCAA, Lopes Neto D. Avaliação de indicadores de rastreamento do câncer do colo do útero no Amazonas, Norte do Brasil, de 2001 a 2005. Revista Brasileira de Cancerologia. 2009;55(3):213-20. Disponível em: http:// www1.inca.gov.br/rbc/n_55/v03/pdf/17_artigo2.pdf

28. Abiodun OA, Olu-Abiodun OO, Sotunsa JO, Oluwole FA. Impact of health education intervention on knowledge and perception of cervical cancer and cervical screening uptake among adult women in rural communities in Nigeria. BMC Public Health. 2014;14(1):814. Disponível em: https://bmcpublichealth.biomedcentral.com/track/ pdf/10.1186/1471-2458-14-814 
29. Corrêa DAD, Villela WV. O controle do câncer do colo do útero: desafios para implementação de ações programáticas no Amazonas. Rev Bras Saúde Matern Infant. 2008;8(4):491-7. Disponível em: http://www.scielo.br/pdf/rbsmi/v8n4/15.pdf

30. Dias MBK, Tomazelli JG, Assis M. Rastreamento do câncer de colo do útero no Brasil: análise de dados do Siscolo no período de 2002 a 2006. Epidemiol Serv Saude. 2010;19(3):293-306. Disponível em: http://scielo.iec.gov.br/pdf/ess/v19n3/v19n3a11.pdf

31. Makkonen $P$, Heinävaara $S$, Sarkeala T, Anttila A. Impact of organized and opportunistic Pap testing on the risk of cervical cancer in young women - A case-control study from Finland. Gynecol Oncol. 2017;147:601-6. Disponível em: https://www.gynecologiconcology-online.net/ article/S0090-8258(17)31335-5/pdf

32. Islami F, Torre LA, Drope JM, Ward EM, Jemal A. Global cancer in women: cancer control priorities. Cancer Epidemiol Biomarkers Prev. 2017;26(4):458-71. Disponível em: http:// cebp.aacrjournals.org/content/cebp/26/4/458.full.pdf

33. Basu P, Ponti A, Anttila A, Ronco G, Senore C, Vale DB, et al. Status of implementation and organization of cancer screening in The European Union Member StatesSummary results from the second European screening report. Int J Cancer. 2017;142:44-56. Disponível em: https://onlinelibrary.wiley.com/doi/epdf/10.1002/ijc.31043

34. Firmino-Machado J, Varela S, Mendes R, Moreira A, Lunet $N$. Stepwise strategy to improve cervical cancer screening adherence (SCAN-Cervical Cancer) - Automated text messages, phone calls and reminders: Population based randomized controlled trial. Prev Med. 2018;114:123-133. Disponível em:https://www.sciencedirect.com/science/article/pii/S0091743518301944

35. Erwin E, Aronson KJ, Day A, Ginsburg O, Macheku G, Feksi $A$, et al. SMS behaviour change communication and eVoucher interventions to increase uptake of cervical cancer screening in the Kilimanjaro and Arusha regions of Tanzania: a randomised, double-blind, controlled trial of effectiveness. BMJ Innov. 2019;0:1-7. Disponível em: https://innovations.bmj.com/content/bmjinnov/early/ 2019/02/22/bmjinnov-2018-000276.full.pdf

36. Moysés RPC. Qualidade de Vida e Eficácia de uma Intervenção Cognitiva em Mulheres Amazônicas em Tratamento do Cancro de Colo de Útero: Um Estudo com Doentes e suas Cuidadoras. [Projeto de Pesquisa- Doutoramento]. Braga (Portugal: Escola de Psicologia da Universidade do Minho; 2017-2020. Disponível em: http://www. saudefamiliaredoenca.pt/investigacao/projdoenca.html 